\title{
Diamond-Gardner Syndrome: Autoerythrocyte Sensitization Syndrome
}

\author{
(1) Nejat Akar ${ }^{1}$, (1) Zeynep Nur Karagöz², (1) Yasemin Ardıçoğlu Akışın³ \\ ${ }^{1}$ TOBB ETU Faculty of Medicine, Department of Paediatrics, Ankara, Turkey \\ 2TOBB ETU Faculty of Medicine, $5^{\text {th }}$ Year Student, Ankara, Turkey \\ ${ }^{3}$ TOBB ETU Faculty of Medicine, Department of Biochemistry, Ankara, Turkey
}

\begin{abstract}
Diamond-Gardner syndrome (DGS) is an autoimmune disease characterized by painful ecchymoses that develop following emotional stress or trauma. The lesions are observed mostly in the extremities and these lesions are the result of autosensitization to extravasated erythrocytes after trauma. The majority of the patients diagnosed with this disease are composed of young women. In this case report, a teenager who had complaints of recurring painful ecchymoses with no related personal or familial background and later-diagnosed with DGS is presented. Although it is seen less commonly, DGS should be considered in the differential diagnosis of cutaneous lesions and haemorrhages of the childhood period, especially in adolescence.
\end{abstract}

Keywords: Diamond-Gardner syndrome, autoerythrocyte sensitization syndrome, Psychogenic purpura

\section{Introduction}

Diamond-Gardner syndrome (DCS) which is also known as autoerythrocyte sensitization syndrome, painful bruising syndrome or psychogenic purpura was first described by Diamond-Gardner in 1955 (1). They discovered recurrent, painful ecchymosis following emotional or physical stress in four women and named the syndrome. In the following years, more cases consisting of ecchymosis which are related with trauma but have no relationship with haematological disorders were reported. The syndrome is mostly seen among adult females but there are also cases in children and adult men $(2,3)$. Although some theories have been put forward, the underlying mechanism of the syndrome remains unclear (4).

In this report, a case of a 16-year-old female patient with DGS is presented.

\section{Case Report}

A 16-year-old female patient was admitted to the paediatric clinics of TOBB ETU Hospital with a complaint of recurrent ecchymosis on both upper and lower extremities.

In her personal history, no other complaints other than ecchymosis which had continued for over 4 months was mentioned. There was no history of epistaxis or menorrhagia. No physical trauma or drug usage was described. Although she denied any emotional trauma, when the matter was more deeply investigated, it was learned that she had been suffering from a drop in success in school. No similar complaints were present among the other family members.

On physical examination, ecchymosis with different diameters and colours were observed, one on the right wrist and two on the shoulders. The one on the right wrist had a $3 \mathrm{~cm}$ diameter and was light pink in colour (Figure 1). 
The one on the left shoulder had a $4 \mathrm{~cm}$ diameter and was purple, the one on the right shoulder was smaller and less prominent than the one on the left shoulder (Figure 2). The ecchymosis was smooth on the surface and was not painful with or without palpation. No other findings other than the ecchymosis were present.

Complete blood count, international normalized ratio, prothrombin time (PT), thrombin time, activated partial thromboplastin time (aPTT), bleeding time and fibrinogen were found to be normal. Antinuclear antibody was found to be negative. On peripheral blood smear, thrombocytes were found sufficient and clustering with no abnormalities.

Since autoerythrocyte sensitization syndrome was considered, the patient was intradermally given $1 \mathrm{~mL}$ of both whole blood and plasma obtained from the patient herself, and also saline was administered to the other arm as a control (Figures 3,4$)$. The skin was examined on the $1^{\text {st }}$

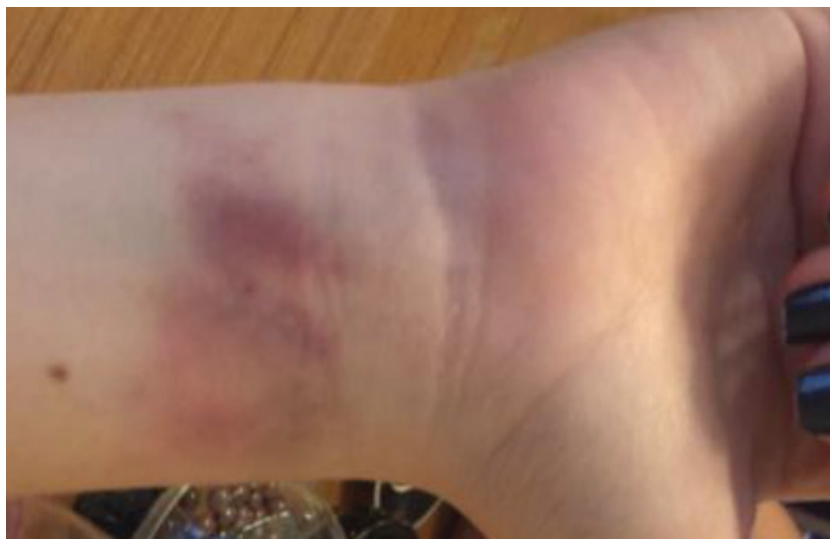

Figure 1. Ecchymosis on the right wrist

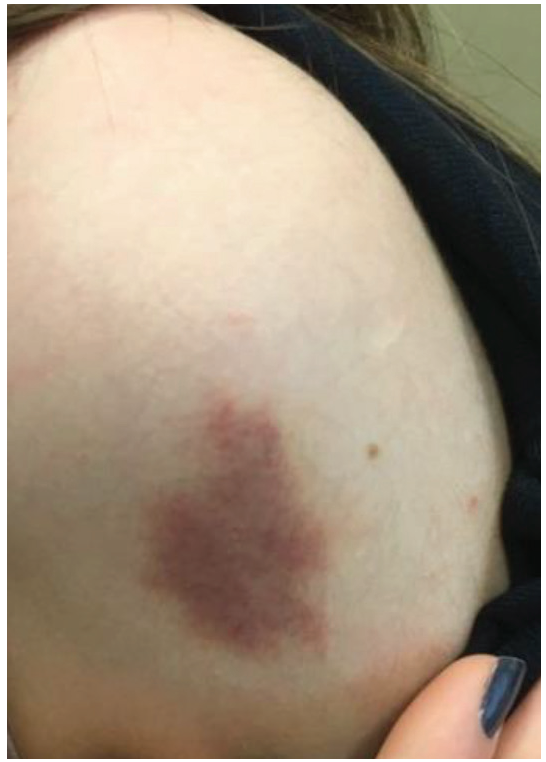

Figure 2. Ecchymosis on the left shoulder and $24^{\text {th }}$ hour after the injection (Figures 3-5). Although the skin reacted to both whole blood and plasma, the reaction to whole blood was more prominent.

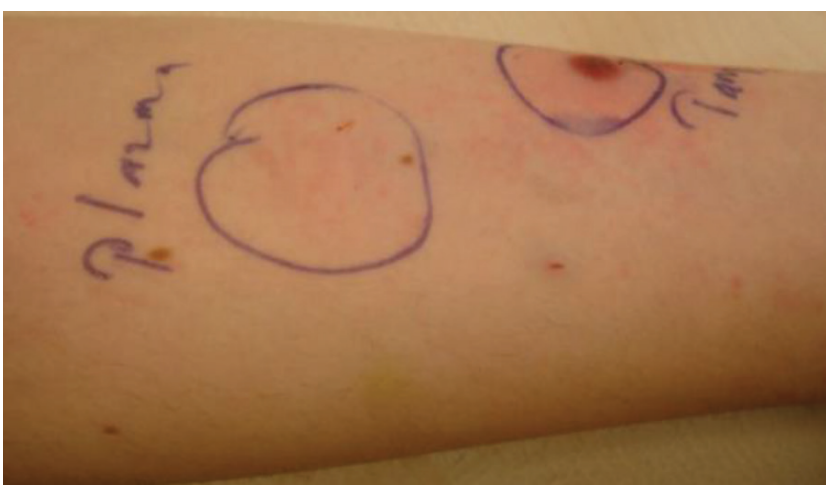

Figure 3. Skin one hour after the injection of plasma and whole blood

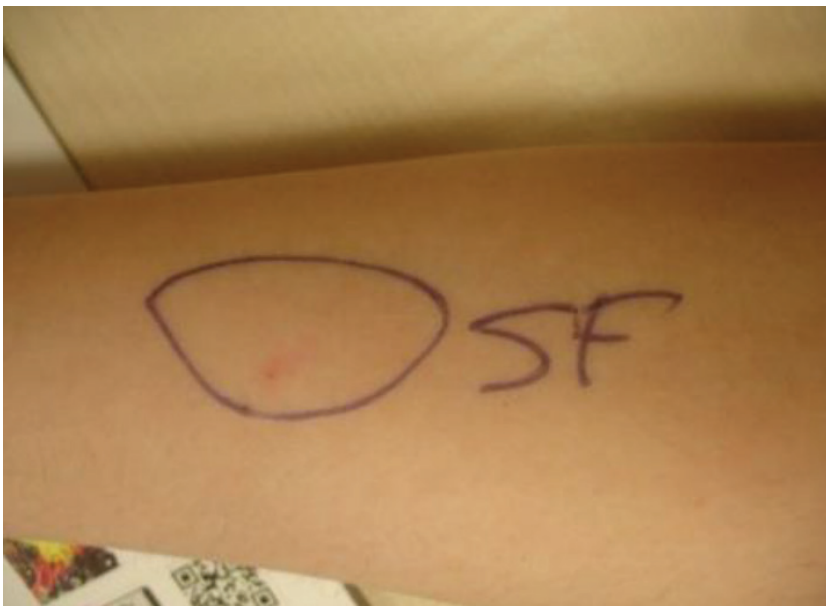

Figure 4. Control injection of saline

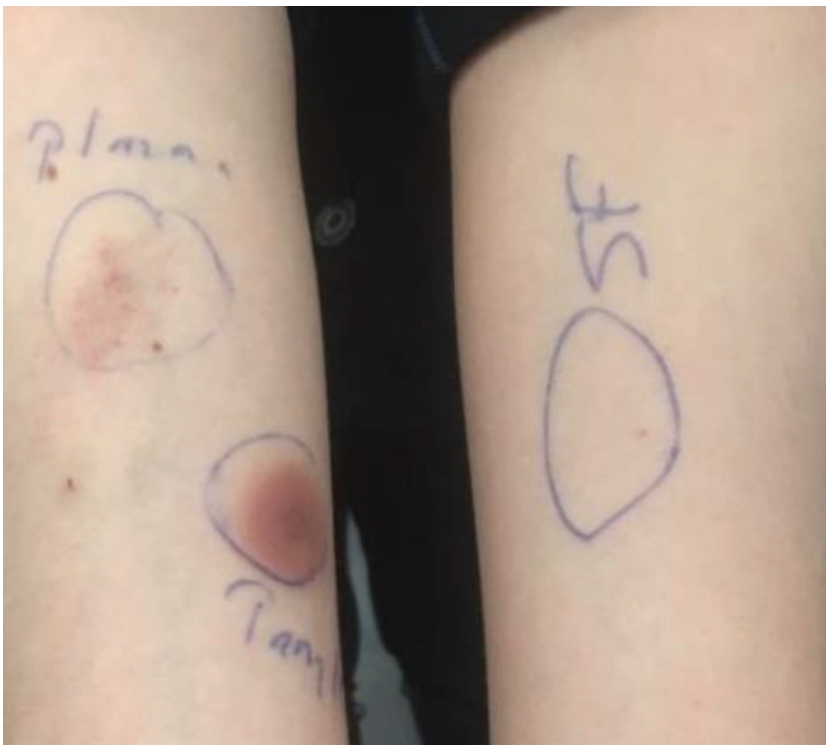

Figure 5. Skin observed 24 hours after the injection 
The pre-diagnosis of DCS was confirmed according to the test result. The patient and her parents consented to the publication of this study.

\section{Discussion}

DGS is a hard-to-diagnose disease which is characterized by recurrent, unexplained bruising that mostly occurs on the extremities and/or face (1). The lesions are found to be well correlated with episodes of increased physical or mental stress (4).

Patients with DGS are vulnerable to trauma and are easily bruised. These bruises result in episodes of ecchymotic lesions which usually begins with burning, pain, and/or tenderness in the affected area. Patients may experience malaise or fatigue and, in some cases, fever, headache, or gastrointestinal symptoms (3). Bruises typically go away approximately in a week and the pain generally lessens when the bruises disappear. However, relapses and remissions of bruising episodes can last during the lifetime of the patient.

The underlying mechanism of this condition is thought to be increased sensitivity to phosphatidylserine in the stroma of the erythrocytes, however it has not been proven yet (4).

There is no specific laboratory test for the diagnosis of this syndrome, diagnosis is made mostly by detailed anamnesis and ruling out the other possible disorders for the lesions. A special skin test in which the patient's own plasma is injected to see the reaction may help in diagnosis, however a negative result of the test does not rule out the syndrome (5).

For the differential diagnosis, factitial purpura, coagulation and platelet disorders, von Willebrand disease, vasculitis and systemic lupus erythematosus should be considered.

Many drugs including antihistaminics, corticosteroids, immunosuppresives, hormones, anticoagulants are used for the treatment of DGS but none of them have been found to be efficient enough. Physiological treatment is seen as the most effective treatment. Although there is no specific treatment, the prognosis of DGS is good, most cases experience recurrent lesions that appear in times of increased stress, but mortality due to the syndrome is not reported.

\section{Ethics}

Informed Consent: The patient and her parents consented to the publication of this study.

Peer-review: External and internal peer-reviewed.

\section{Authorship Contributions}

Surgical and Medical Practices: N.A., Concept: N.A., Design: N.A., Data Collection or Processing: Y.A.A., Analysis or Interpretation: Y.A.A., Literature Search: Z.N.K., Writing: Z.N.K.

Conflict of Interest: No conflict of interest was declared by the authors.

Financial Disclosure: The authors declared that this study received no financial support.

\section{References}

1. Gardner FH, Diamond LK. Autoerythrocyte sensitization: a form of purpura producing painful bruising following autosensitization to red blood cells in certain women. Blood 1995;10:675-90.

2. Ingber A, Alcalay ), Feuerman E). Autoerythrocyte sensitization (Gardner-Diamond syndrome) in men: a case report and review of the literature. Postgrad Med I 1985;61:823-6.

3. Gözdaşoğlu, S. Autoerythrocyte Sensitization Syndrome Treated with Kallikrein Inhibitor. Turk J Hematol 2013;30:96-7.

4. Ratnoff OD. Psychogenic purpura (autoerythrocyte sensitization): An unsolved dilemma. Am / Med 1989;87:16N$21 \mathrm{~N}$.

5. Okur M, Turan H, Özkan A, Güneş C, Kocabay K. An Extremely Rare Cause of Bruising in Children: Autoerythrocyte Sensitization Syndrome. Turk J Hematol 2012;29:201-3. 